\title{
Genome assembly and annotation of Macadamia tetraphylla
}

\author{
Ying-Feng $\mathrm{Niu}^{1 *}$, Guo-Hua $\mathrm{Li}^{1}$, Shu-Bang $\mathrm{Ni}^{1}$, Xi-Yong He${ }^{1}$, Cheng Zheng ${ }^{1}$, Zi-Yan Liu ${ }^{1}$, Li-Dan \\ Gong ${ }^{1}$, Guang-Hong Kong ${ }^{1}$, Jin Liu ${ }^{1 *}$ \\ ${ }^{1}$ Yunnan Institute of Tropical Crops, Xishuangbanna 666100, China. \\ *These authors contributed equally to this work. \\ Correspondence and requests for materials should be addressed to Jin Liu (liujin06@126.com).
}

\begin{abstract}
Macadamia is a kind of evergreen nut trees which belong to the Proteaceae family. The two commercial macadamia species, Macadamia integrifolia and M. tetraphylla, are highly prized for their edible kernels. Catherine et al. reported M. integrifolia genome using NGS sequencing technology. However, the lack of a high-quality assembly for $M$. tetraphylla hinders the progress in biological research and breeding program. In this study, we report a high-quality genome sequence of M. tetraphylla using the Oxford Nanopore Technologies (ONT) technology. We generated an assembly of $750.54 \mathrm{Mb}$ with a contig N50 length of $1.18 \mathrm{Mb}$, which is close to the size estimated by flow cytometry and k-mer analysis. Repetitive sequence represent $58.57 \%$ of the genome sequence, which is strikingly higher compared with $M$. integrifolia. A total of 31,571 protein-coding genes were annotated with an average length of $6,055 \mathrm{bp}$, of which $92.59 \%$ were functionally annotated. The genome sequence of $M$. tetraphylla will provide novel insights into the breeding of novel strains and genetic improvement of agronomic traits.

Key words: Macadamia tetraphylla, Genome, Assembly, Annotation
\end{abstract}

\section{Background \& Summary}

Macadamia is a kind of evergreen nut trees which belong to the Proteaceae family, Macadamia F. Muell. genus, grown commercially for their high-value kernels ${ }^{1}$. The Macadamia F. Muell. genus contains four different species, namely M. integrifolia, M. tetraphylla, M. ternifolia \& M. jansenii ${ }^{2}$, but only M. integrifolia, M. tetraphylla and the hybrids of them (Macadamia integrifolia $\times$ Macadamia tetraphylla) were used to 
produce nuts ${ }^{3-5}$. Although macadamia is native to the subtropical rainforest in Queensland and New South Wales of Australia ${ }^{6,7}$, but its large-scale commercial cultivation was began in Hawaii of America in $1948^{8}$, so it's also known as Hawaiian Nuts.

The kernel of macadamia are rich in unsaturated fatty acids, essential amino acids, Trace elements and vitamins, the content of monounsaturated fatty acids and palmitoleic acid are also extremely rich $^{9-11}$. Macadamia nuts can be eaten directly, also can be used as raw material for processing high-grade edible oil ${ }^{12}$. Because it is very nutritious, long-term consumption of macadamia helps to lower blood cholesterol, prevent arteriosclerosis, lowering the viscosity of platelets, reduce the incidence of heart disease, myocardial infarction and other cardiovascular diseases ${ }^{13}, 14$, therefore, the macadamia nuts were favored by consumers, known as the "Queen of nuts."

World consumption of macadamia nuts rapid growth in recent years, according to the FAO statistics and projections, the current world macadamia total demand is more than 400,000 tons, while the supply is only about 40,000 tons, within the current and future long period of time, macadamia nuts production is still in short supply.

Macadamia commercial growing areas located mainly in tropical and subtropical areas ${ }^{15}$, the traditional cultivated area is the United States and Australia ${ }^{16}$. Because macadamia cultivation technology simple, planting high income, more cold-resistant than rubber trees, bananas and other traditional tropical crops, very suitable for some slightly cold area of tropical and subtropical countries, so the planted area growth rapidly over the past decade. However, currently the world's macadamia orchard area in descending order are China, South Africa, Australia, Kenya, Guatemala and United States, Which China's plantation area has reached 300,000 hectares, is the world's largest country of Macadamia acreage.

In recent years, genome sequencing of many important tropical crops have been reported, although macadamia are diploid $(2 \mathrm{n}=28)$ with genome size estimates ranging from 652 $\mathrm{Mb}$ to $780 \mathrm{Mb}^{17-19}$, but research on the genome of macadamia was very few. In 2014, 2017 and 2018, the chloroplast genome of M. integrifolia, M. ternifolia and $M$. tetraphylla have been sequenced by Australian and Chinese researchers ${ }^{20-22}$, and in 2016, the darft genome and transcriptome of $M$. integrifolia cultivar 741 have been sequenced, a total length of $518 \mathrm{Mb}$ have been assembly, which spans approximately $79 \%$ of the 
estimated genome $\operatorname{size}^{18}$. But up to now, the genome sequencing of M. tetraphylla has not yet been reported. As an important parent species of macadamia varieties in production ${ }^{23}$, the genome sequencing of $M$. tetraphylla will lay a good foundation for macadamia breeding.

\section{Methods}

\section{Sample collection, library construction and sequencing}

A cultivated individual of $M$. tetraphylla was collected from Xishuangbanna, Yunnan Province, China. The collected plant samples were immediately frozen in liquid nitrogen and stored at $-80^{\circ} \mathrm{C}$ before DNA isolation. High-molecular-weight genomic DNA was extracted using Qiagen plant genomic DNA extraction kit. The extracted DNA was prepared for sequencing following the manufacturer's instructions in the genomic sequencing kit SQK-LSK108. The library was loaded on a single R9.4 flowcell and then sequenced on a GridION X5 platform. A total of $68.17 \mathrm{~Gb}$ data were generated with an average read length of $20.22 \mathrm{~kb}$ (Table 1 and Fig. 1a). We also generated $88.27 \mathrm{~Gb}$ short reads on the HiSeq2500 platform (Illumina, San Diego, CA, USA) with an insert size of $500 \mathrm{bp}$ for the purpose of genome survey and assembly polishing (Table 2).

Table 1. Statistics of Oxford nanopore long-reads used for genome assembly

\begin{tabular}{lllll}
\hline $\begin{array}{l}\text { Pass Reads } \\
\text { Number }\end{array}$ & $\begin{array}{l}\text { Pass Reads Bases } \\
\text { (bp) }\end{array}$ & $\begin{array}{l}\text { Mean Reads } \\
\text { Length (bp) }\end{array}$ & $\begin{array}{l}\text { Reads N50 } \\
\text { Length (bp) }\end{array}$ & $\begin{array}{l}\text { Mean Read } \\
\text { Quality }\end{array}$ \\
\hline $3,382,098$ & $68,173,294,275$ & 20,157 & 24,256 & 7.6 \\
\hline
\end{tabular}

Table 2. Statistics of Illumina sequencing reads used for genome and transcriptome assembly

\begin{tabular}{lllll}
\hline Data Type & Library & Insert Size (bp) & Read Length (bp) & Total Bases (Gb) \\
\hline DNA & PE01 & $500 \mathrm{bp}$ & 150 & 88.27 \\
\hline \multirow{4}{*}{ RNA } & T01 & $250 \mathrm{bp}$ & 150 & 6.49 \\
& T02 & $250 \mathrm{bp}$ & 150 & 6.58 \\
& T03 & $250 \mathrm{bp}$ & 150 & 8.55 \\
& T04 & $250 \mathrm{bp}$ & 150 & 6.40 \\
& T05 & $250 \mathrm{bp}$ & 150 & 6.28 \\
\hline
\end{tabular}

Total RNA was isolated from five tissues (young leaves, young inflorescences, flowering 
inflorescence, proteoid root, and bark) using the Column Plant RNAout kit (TIANDZ, China). All cDNA libraries were prepared using an Illumina TruSeq RNA Library Prep Kit and sequenced on HiSeq2500 platform. A total of $34.3 \mathrm{~Gb}$ clean data were obtained after quality control (Table 2).
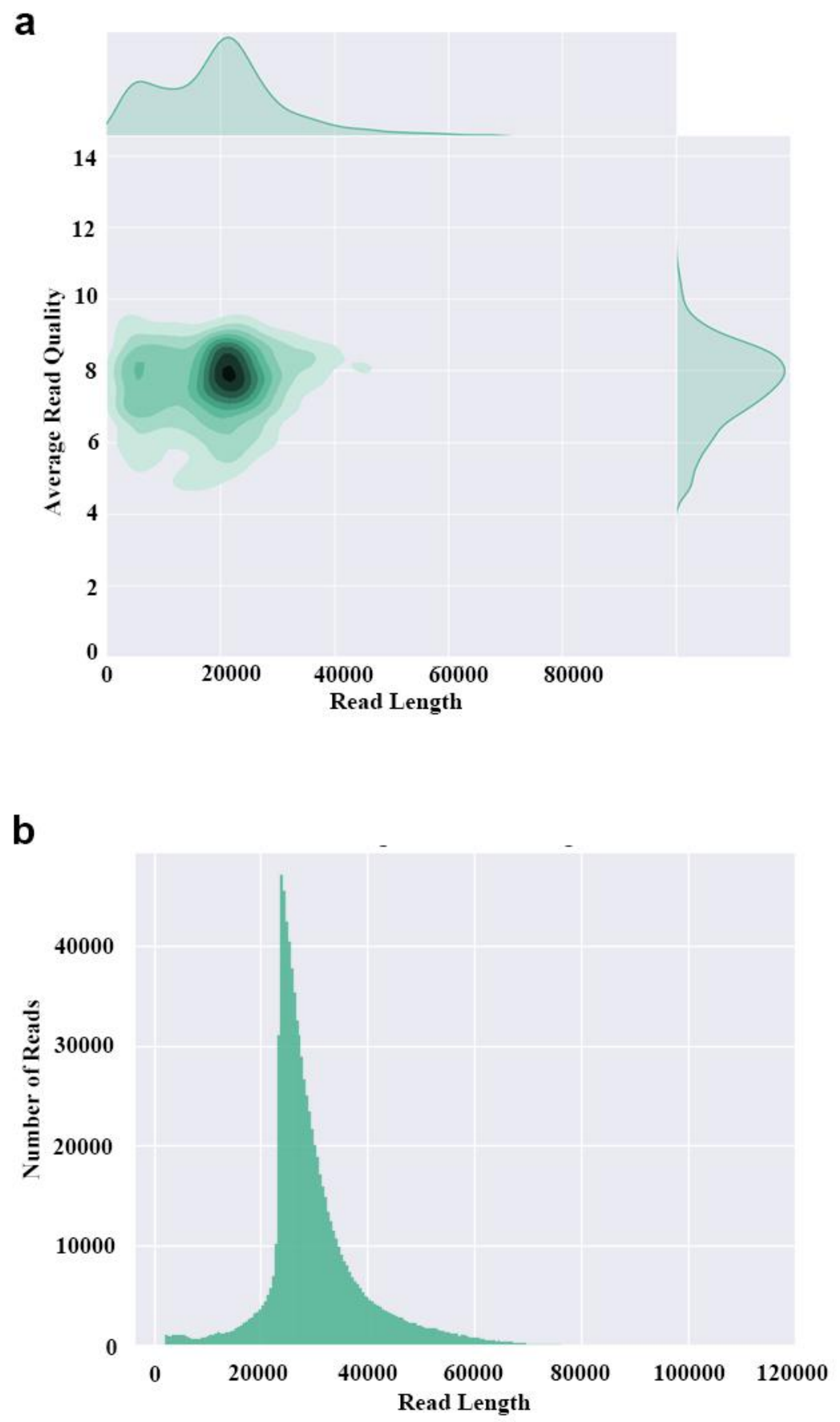

Figure 1. Length distribution of Oxford nanopore long reads. (a) Read length and read quality of the original reads; (b) Read length of the corrected reads. 


\section{Estimation of genome size and heterozygosity}

The genome size of $M$. tetraphylla was estimated by flow cytometry following the protocol described by Dolezel ${ }^{24}$ and the deduced genome size was $740 \mathrm{Mb}$. We further evaluated the genome size by performing k-mer frequency analysis. In brief, Jellyfish $\mathrm{v} 2.1 .0^{25}$ was used to generate the 17-mer frequency distribution of paired end reads, the genome size was estimated according to the formula: $G=K \_$num/peak depth (G: genome size; K_num: total number of k-mers; peak depth: depth of the major peak). The cumulative k-mer count suggested the genome size be $758 \mathrm{Mb}$ (Fig. 2), which is similar to the result generated by flow cytometry. We estimated the heterozygosity level of the $M$. tetraphylla genome to be $1.03 \%$ using GenomeScope ${ }^{26}$.

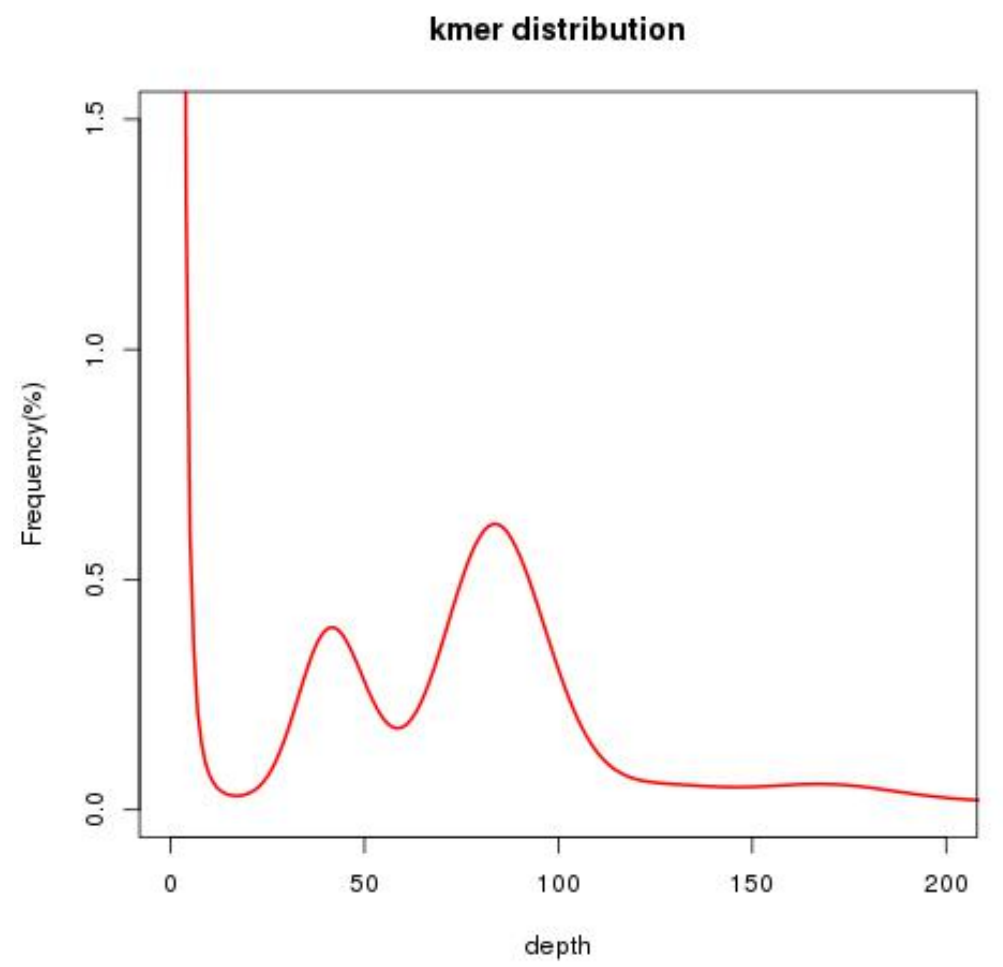

Figure 2. The 17-mer distribution of Illumina sequencing reads from the M. tetraphylla genome.

\section{Genome assembly}

A hybrid assembly pipeline was used to alleviate the effects of the highly heterozygous genome with highly repetitive DNA sequences (Fig. 3). The nanopore raw reads were corrected and trimmed using Canu v1.827. A total of $26.34 \mathrm{~Gb}$ clean data was obtained with an average length of $29.89 \mathrm{~kb}$ (Fig. 1b). The corrected reads were then fed to 
WTDBG v2.2 $2^{28}$ for genome assembly with the following parameters: -S 2 --edge-min 2 --rescue-low-cov-edges -x ccs -g 800m. Iterative polishing was performed using Pilon $\mathrm{v} 1.23^{29}$ to fix bases, fill gaps, and correct local misassemblies. Finally, we assembled the M. tetraphylla genome into 4,335 contigs, with an N50 of 1,182,547 bp (Table 3). The assembly size $(750 \mathrm{Mb})$ was consistent with the estimated genome size based on flow cytometry and k-mer analysis (740 Mb and $758 \mathrm{Mb}$, respectively).

Table 3. Comparison between genome assemblies for two macadamia species.

\begin{tabular}{lll}
\hline Genome features & M. tetraphylla & M. integrifolia \\
\hline Estimated genome size $(\mathrm{Mb})$ & 758 & 652 \\
Total length of assembly (bp) & $750,539,052$ & $518,489,877$ \\
No. of contigs & 4,335 & 210,726 \\
Contig N50 (bp) & $1,182,547$ & 3,522 \\
Longest contig (bp) & $9,780,764$ & 379,349 \\
No. of scaffolds & 4,335 & 193,493 \\
Scaffold N50 (bp) & $1,182,547$ & 4,745 \\
Longest scaffold (bp) & $9,780,764$ & 643,490 \\
\hline
\end{tabular}

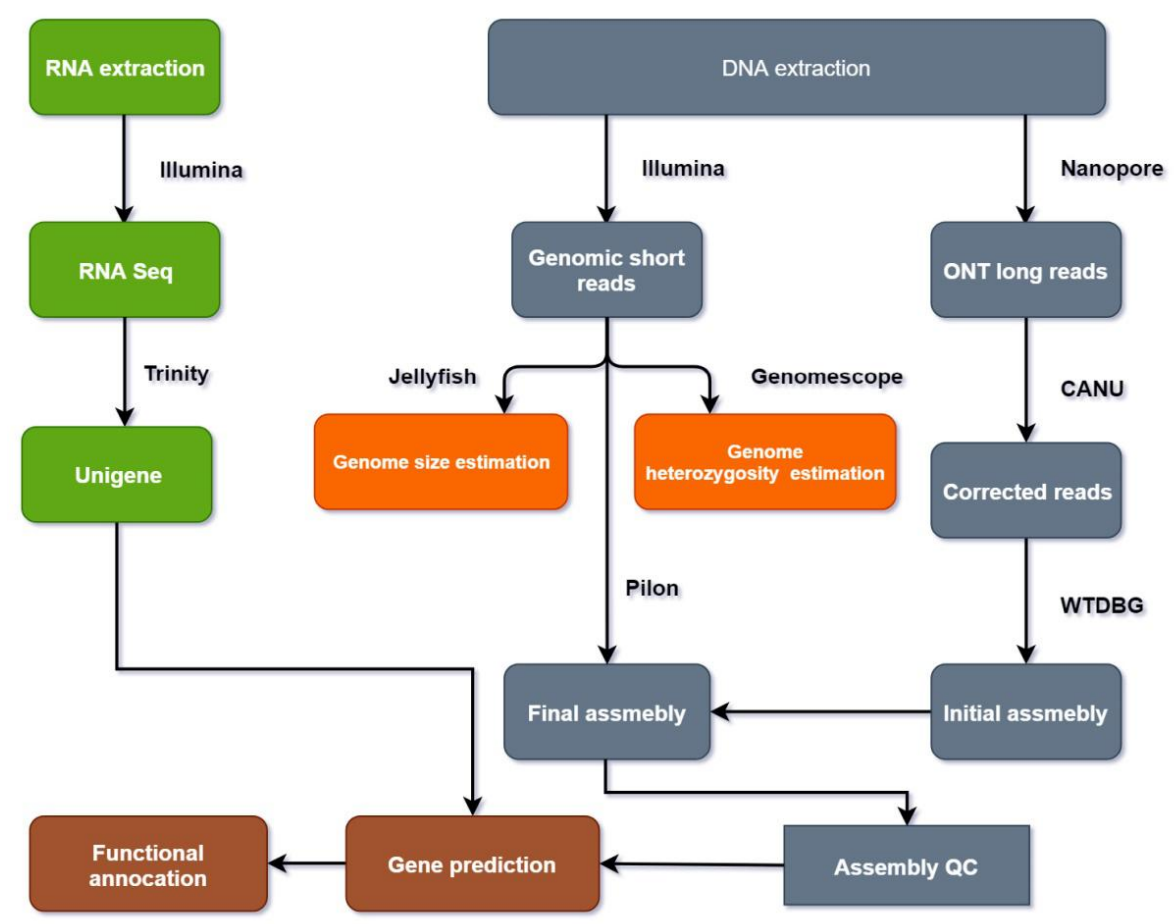

Figure 3. Overview of the pipeline used in this study 


\section{Genome annotation}

Repeat annotation. Two complementary methods were used to identity repetitive sequences in the M. tetraphylla genome. First, Tandem Repeats Finder v4.09 ${ }^{30}$ was used to identity the tandem repeats. Next, a combined strategy was selected to predict transposable elements (TEs). For homology-based annotation of TEs, RepeatMasker v1.332 (http://www.repeatmasker.org) was employed to search against RepBase database $(\mathrm{v} 18.07)^{31}$, and RepeatProteinMasker ${ }^{32}$ was used to search the protein database to filter TE-related proteins. Three softwares, including RepeatModeler v1.05 (http://www.repeatmasker.org/RepeatModeler.html), RepeatScout v1.05 ${ }^{33}$, and Piler $\mathrm{v} 1.06^{34}$, were used to construct a de novo library. RepeatMasker was then applied for comprehensive identification of TEs. A total of $461 \mathrm{Mb}$, which represents $61.42 \%$ of the genome (Table 4), were identified as repeats, much higher than that in $M$. integrifolia genome (37\%). Long terminal repeat (LTR) retrotransposons represent the most predominant class of transposable elements. Result showed that M. tetraphylla contains $34.95 \%$ LTR retrotransposons, of which $22.00 \%$ are Gypsy-type elements and 5.94\% are Copia-type elements (Table 4).

Table 4. Annotation of repeat sequences in the M. tetraphylla genome

\begin{tabular}{|c|c|c|c|}
\hline Repeat elements & Copy number & Length (bp) & Percentage $(\%)$ \\
\hline Retrotransposons & 214,430 & $262,347,793$ & 34.95 \\
\hline Non-LTR retrotransposons & 72,628 & $44,629,376$ & 5.95 \\
\hline LTR retrotransposons & 141,802 & $217,718,417$ & 29.01 \\
\hline Gypsy & 88,052 & $165,151,480$ & 22.00 \\
\hline Copia & 46,649 & $44,586,669$ & 5.94 \\
\hline Other & 7,101 & $7,980,268$ & 1.06 \\
\hline DNA transposons & 11,167 & $7,521,183$ & 1.00 \\
\hline Unclassified elements & 947,252 & $191,137,916$ & 25.47 \\
\hline Total & $1,172,849$ & $461,006,892$ & 61.42 \\
\hline
\end{tabular}

Simple sequence repeats (SSRs) were identified in the $M$. tetraphylla genome using MISA program ${ }^{35}$, with the following parameters: at least twelve repeats for monomer, six repeats for dimer, four repeats for trimer, three repeats for tetramer, pentamer and 
hexamer. A total of 510,893 SSRs were identified in M. tetraphylla genome (Table 5). Among the repeat motifs, mono-nucleotide repeats were the most predominant (Table 5), followed by di-, tri-, tetra-, penta- and hexa-nucleotide (Fig. 4 and Table 5). All these identified SSR markers may serve as potential markers of interest to M. tetraphylla breeding programs.

a
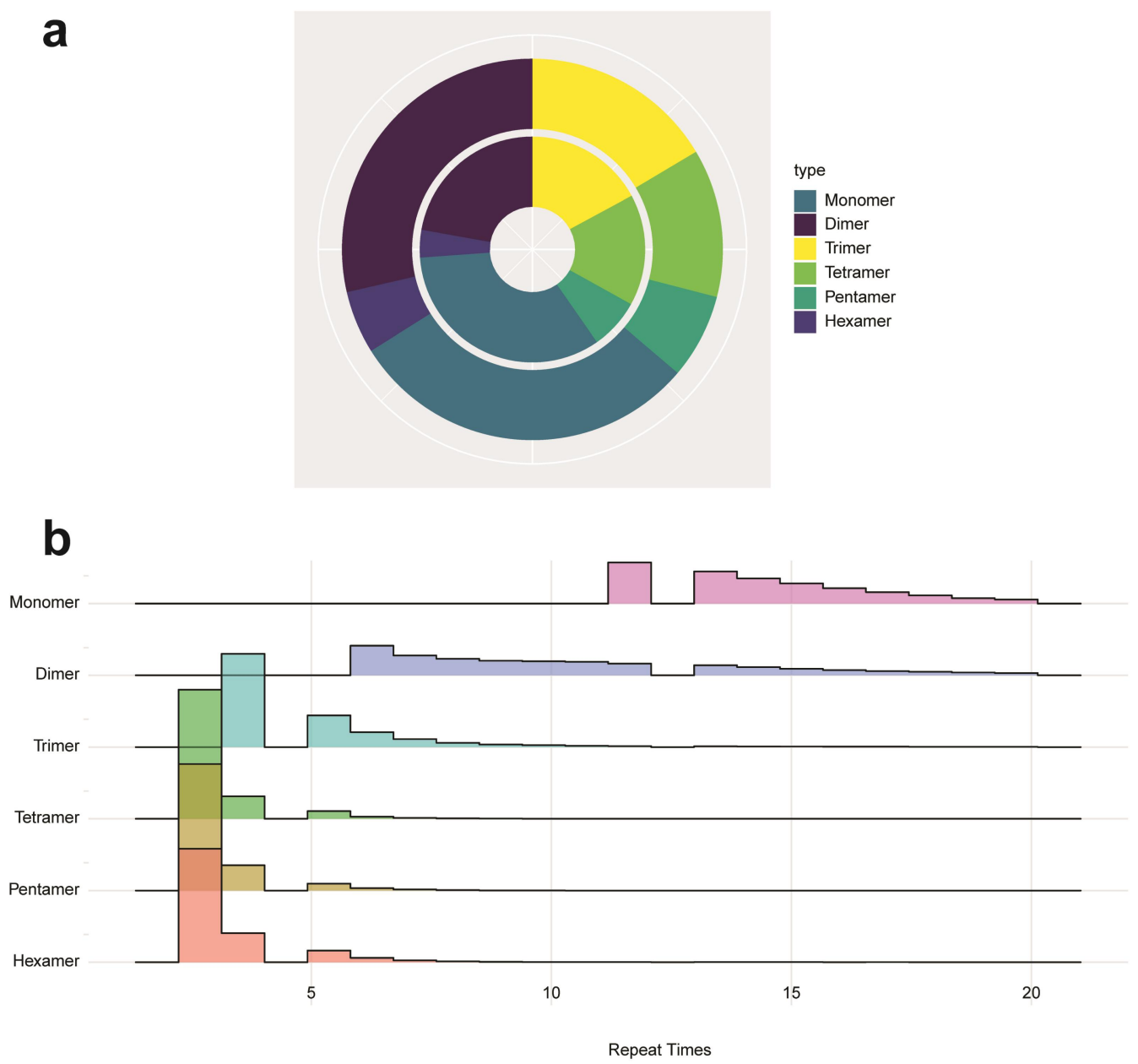

Figure 4. Summary of SSRs detected in M. tetraphylla genome. (a) Proportion of different types of SSRs. The outer circle represents the length proportion and the inner circle represents the number proportion; (b) Repeat times distribution of SSRs

Noncoding RNA. Five types of non-coding RNA genes, tRNA, rRNA, snRNA, snoRNA and miRNA, were identified in the $M$. tetraphylla genome. The tRNA genes were identified using tRNAscan-SE v2.036. For identification of rRNA, RNAmmer v1.2 $2^{37}$ was 
used to screen against the genome sequence. The snRNA genes were predicted using INFERNAL software $(\mathrm{v} 1.1 .2)^{38}$ with $\mathrm{cm}$ models from Rfam database ${ }^{39}$. The snoRNA genes were identified by adopting snoscan v0.9.140. A total of 1,286 tRNAs, 542 rRNAs, 251 snRNAs, and 74 snoRNAs were identified in M. tetraphylla genome (Table 6).

Table 5. Summary of simple sequence repeats (SSRs) identified in the M. tetraphylla genome

\begin{tabular}{llll}
\hline Type & Unit size & Number & Length (bp) \\
\hline Monomer & $\mathrm{n}>=12$ & 171,372 & $2,565,684$ \\
Dimer & $\mathrm{n}>=6$ & 113,278 & $2,454,290$ \\
Trimer & $\mathrm{n}>=4$ & 87,467 & $1,412,550$ \\
Tetramer & $\mathrm{n}>=3$ & 81,444 & $1,079,288$ \\
Pentamer & $\mathrm{n}>=3$ & 37,088 & 617,310 \\
Hexamer & $\mathrm{n}>=3$ & 20,244 & 456,330 \\
Total Number & & 510,893 & $8,585,452$ \\
\hline
\end{tabular}

Table 6. Annotation of non-coding RNA genes identified in the M. tetraphylla genome

\begin{tabular}{lllll}
\hline Type & Number & Average length (bp) & Total length (bp) & Perceentage (\%) \\
\hline tRNA & 1,286 & 74.34 & 95,604 & 0.0127 \\
rRNA (8s) & 210 & 112.94 & 23,718 & 0.0032 \\
rRNA (18s) & 177 & $1,886.61$ & 333,931 & 0.0445 \\
rRNA (28s) & 155 & $4,333.59$ & 671,707 & 0.0895 \\
snRNA & 251 & 122.01 & 30,624 & 0.0041 \\
snoRNA & 74 & 114.12 & 8,445 & 0.0011 \\
\hline
\end{tabular}

Gene prediction and functional annotation. Gene prediction was carried out combining de novo, homology, and EST-based predictions. Augustus v2.741 and SNAP version 2006-07-28 $8^{42}$ were used to perform de novo prediction. The assembled transcripts generated in this study were used for iteratively self-training, and the optimized parameters were applied for further annotation by Augustus and SNAP. For homology prediction, protein sequences from Arabidopsis thaliana ${ }^{43}$, Malus domestica ${ }^{44}$, Nelumbo nucifera ${ }^{45}$, and Rosa chinensis ${ }^{46}$ were aligned to the genome using genblastA v1.0.147. The hits regions were extended in both $3^{\prime}$ and $5^{\prime}$ directions and fed to GeneWise v2.2.0 ${ }^{48}$ 
to obtain accurate spliced alignments. The transcripts were also mapped to the genome to generate spliced alignments using Program to Assemble Spliced Alignments (PASA)

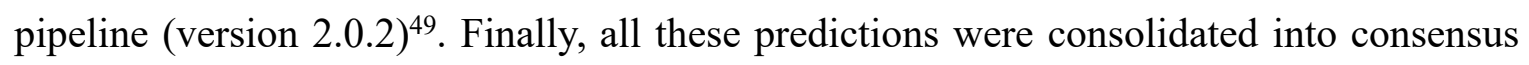
gene set using EVidenceModeler (r2012-06-25) ${ }^{50}$. In this way, we identified 31,571 genes, with an average length of 6,055 bp and an average CDS length of 1,123 bp (Table 7).

Table 7. Prediction of protein-coding genes identified in the M. tetraphylla genome

\begin{tabular}{ll}
\hline Gene features & \\
\hline Total number of protein-coding genes & 31,571 \\
Gene length in genome (\%) & 25.47 \\
Average gene length (bp) & 6,055 \\
Average exon length (bp) & 222 \\
Average CDS length (bp) & 1,123 \\
Average intron length (bp) & 1,213 \\
Average exons per gene & 5.1 \\
Gene density (gene per Mb) & 42.04 \\
\hline
\end{tabular}

Functional assignment was carried out by performing blastp searches (with e value 1e-5) against SwissProt database ${ }^{51}$. The KAAS server ${ }^{52}$ was used to map the predicted genes onto KEGG metabolic pathways. InterProScan v5.10-50.0 ${ }^{53}$ determined the motifs and functional domain. The GO term and pfam domain were directly obtained from the InterProScan results. A total of 29,233 genes (92.59\%) were functionally annotated by these methods (Fig. 5 and Table 8).

Table 8. Functional annotation of the protein-coding genes

\begin{tabular}{lll}
\hline Database & Number & Percentage (\%) \\
\hline Swiss-Prot & 22,869 & 72.44 \\
KEGG & 8,303 & 26.30 \\
InterPro & 29,052 & 92.02 \\
GO & 17,864 & 56.58 \\
Pfam & 21,925 & 69.45 \\
Total & 29,233 & 92.59 \\
\hline
\end{tabular}




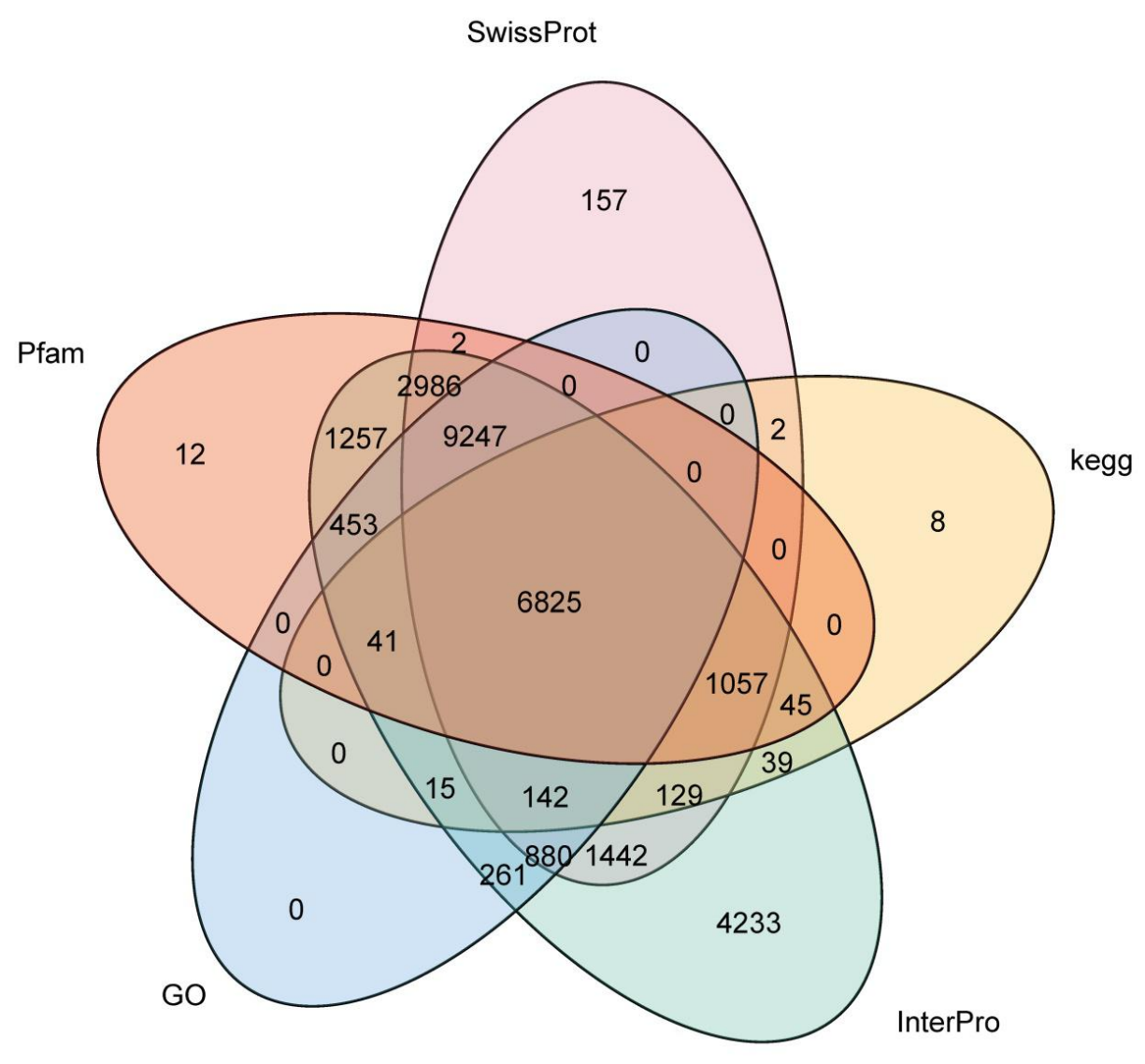

Figure 5. Venn diagram of functional annotation

\section{Data Records}

All sequencing Illumina genomic reads and Oxford nanopore long reads have been deposited in the NCBI Sequence Read Archive (SRA) under the accession number SAMN13219572. RNA-seq reads of five tissues were also available at NCBI SRA database with the accession number SAMN13241873, SAMN13241875, SAMN13241876, SAMN13241877 and SAMN13241873.

\section{Technical Validation}

Quality filtering of Illumina short reads. The Illumina raw reads were trimmed using Trimmomatic $\mathrm{v} 0.32^{54}$ for quality. The adaptors were removed, low-quality and $\mathrm{N}$ base reads were trimmed. The reads were cut when the average quality per base drops below 15 with a 4-base wide sliding window. 
Evaluation of genome assembly. To assess the completeness and accuracy of the genome assembly, the Illumina sequencing reads were mapped to the genome using bowtie 2 v2.2.6 $6^{55}$. Result showed that $94.25 \%$ of the short reads could be mapped to the genome, with an $87.84 \%$ properly paired mapping rate (Table 9). Additionally, the assembly was evaluated by BUSCO (Benchmarking Universal SingleCopy Orthologs) ${ }^{56}$. About $89.72 \%$ ( 1,292 out of the 1,440) conserved genes in embryophyta lineage were present in the assembly (Table 9). To further evaluate the genome assembly, the RNA reads were mapped to the genome using HISAT2 ${ }^{57}$ with a $92.00 \%$ success rate (Table 9). We have also calculated the GC content with a $2 \mathrm{~kb}$ non-overlapping sliding window and there is no obvious GC bias in the genome assembly (Fig. 6). All these results suggested a high quality of $M$. tetraphylla genome.
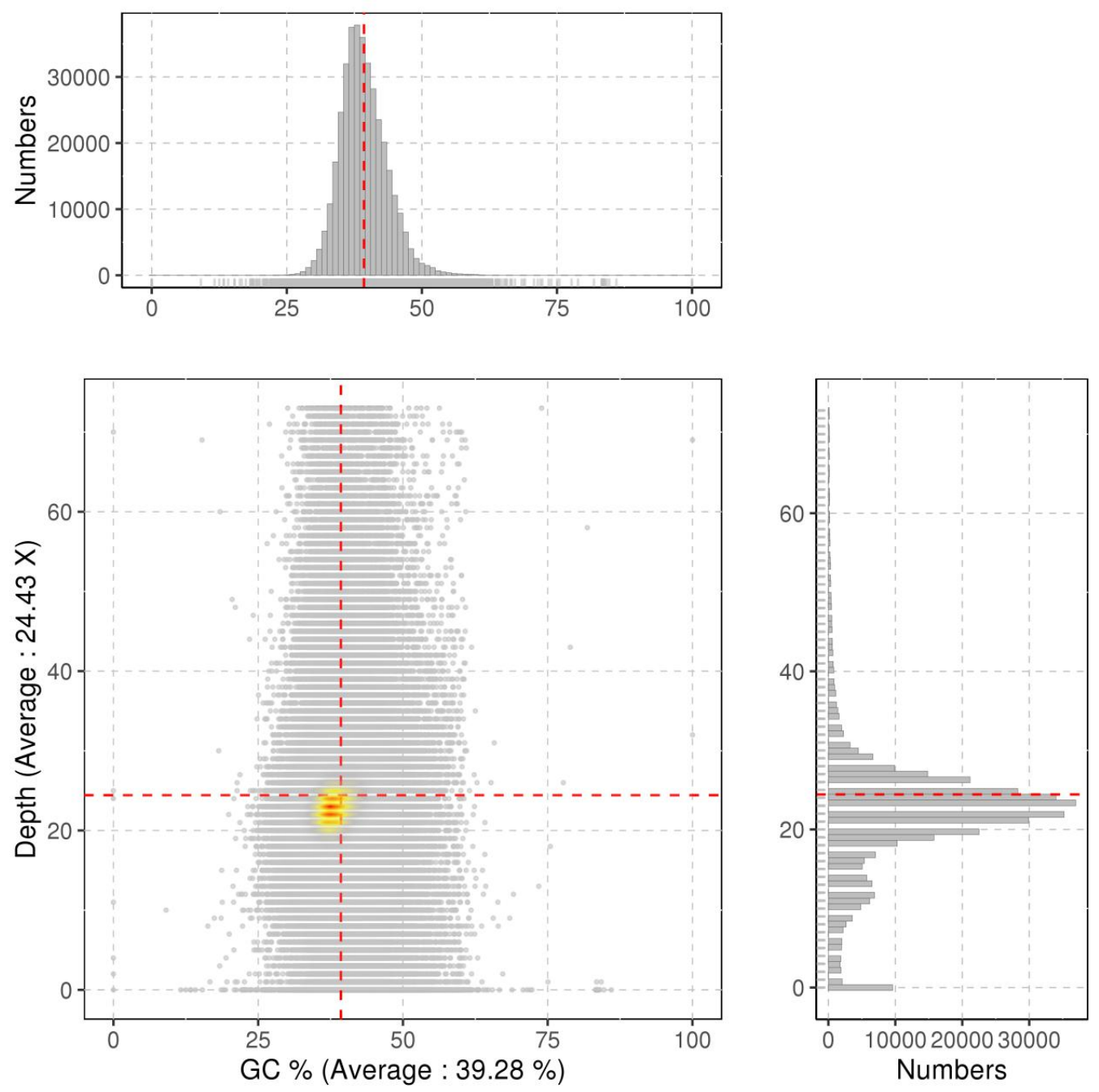

Figure 6. The GC content distribution of the M. tetraphylla genome 
Table 9. Evaluation of genome assembly using different methods

\begin{tabular}{lll}
\hline Method & Feature & Value \\
\hline Reads mapping & Reads number & $573,843,844$ \\
& Mapped number & $540,860,068$ \\
& Mapping rate (\%) & 94.25 \\
& Properly mapped rate (\%) & 87.84 \\
\hline BUSCO & Complete number & 1250 \\
& Fragmented number & 42 \\
\hline RNA-Seq & Missing rate (\%) & 10.3 \\
& Reads number & $146,233,111$ \\
& Mapped number & $134,531,219$ \\
& Mapping rate (\%) & 92.00 \\
\hline
\end{tabular}

Code Availability. The execution of this work involved many software tools, whose versions, settings and parameters are described below.

(1) Jellyfish: version 2.1.0, -m =17; (2) GenomeScope: $-\mathrm{k}=17$; (3) Canu: version 1.8, correctedErrorRate $=0.050$; (4) WTDBG: version $2.2, \quad$-S 2 --edge-min 2 --rescue-low-cov-edges -x ccs -g 800m; (5) Pilon: version 1.23, default parameters; (6) Tandem Repeats Finder: version 4.09, default parameters; (7) RepeatMasker: version 1.332, e ncbi -pa 20; (8) RepeatProteinMasker: default parameters; (9) RepeatModeler: version 1.05, -engine ncbi -pa 20; (10) RepeatScout: version 1.05, default parameters; (11) Piler: version 1.06, default parameters; (12) MISA: 1-12 2-6 3-4 4-3 5-3 6-3; (13) tRNAscan-SE: version 2.0, default parameters; (14) RNAmmer: version 1.2, -S euk -m 1su,ssu,tsu -f rRNA.fasta -gff rRNA.gff2 -h rRNA.hmmreport; (15) INFERNAL: version 1.1.2, default parameters; (16) snoscan: version 0.9.1, default parameters; (17) Augustus: version 2.7, --gff3=on; (18) SNAP: version 2006-07-28, default parameters; (19) genblastA: version 1.0.1, -e 1e-5 -c 0.5 -d 60000; (20) GeneWise: version 2.2.0, default parameters; (21) PASA: version 2.0.2, default parameters; (22) EVidenceModeler: version r2012-06-25, default parameters; (23) blastp: version 2.2.26, default parameters; (24) InterProScan: version v5.10-50.0, -goterms; (25) Trimmomatic: version 0.32, LEADING:3 TRAILING:3 SLIDINGWINDOW:4:15 MINLEN:36; (26) bowtie2: 
version 2.2.6, default parameters; (27) Trinity: version 2.8.4, --seqType fq --max_memory 600G --no_normalize_reads --full_cleanup --min_contig_length 250; (28)

HISAT2: default parameters.

\section{References}

1. Toft, B.D., Alam, M. \& Topp, B. Estimating genetic parameters of architectural and reproductive traits in young macadamia cultivars. Tree Genetics \& Genomes 14, 50 (2018).

2. Akinsanmi, O.A., Neal, J., Drenth, A. \& Topp, B. Characterisation of accessions and species of Macadamia to stem infection by Phytophthora cinnamomi. Plant Pathology 66, 186-193 (2017).

3. Hardner, C. Macadamia domestication in Hawai 'i. Genetic resources and crop evolution $\mathbf{6 3}$, 1411-1430 (2016).

4. Howlett, B.G., Nelson, W.R., Pattemore, D.E. \& Gee, M. Pollination of macadamia: Review and opportunities for improving yields. Scientia Horticulturae 197, 411-419 (2015).

5. Sedgley, M. Pollen tube growth in macadamia. Scientia Horticulturae 18, 333-341 (1983).

6. Neal, J.M., Hardner, C.M. \& Gross, C. Population demography and fecundity do not decline with habitat fragmentation in the rainforest tree Macadamia integrifolia (Proteaceae). Biological Conservation 143, 2591-2600 (2010).

7. Shapcott, A. \& Powell, M. Demographic structure, genetic diversity and habitat distribution of the endangered, Australian rainforest tree Macadamia jansenii help facilitate an introduction program. Australian Journal of Botany 59, 215-225 (2011).

8. Ahmad Termizi, A. et al. in XXIX International Horticultural Congress on Horticulture: Sustaining Lives, Livelihoods and Landscapes (IHC2014): 1109 175-180 (2014).

9. Kaijser, A., Dutta, P. \& Savage, G. Oxidative stability and lipid composition of macadamia nuts grown in New Zealand. Food Chemistry 71, 67-70 (2000).

10. Malvestiti, R. et al. The effect of macadamia oil intake on muscular inflammation and oxidative profile kinetics after exhaustive exercise. European journal of lipid science and technology 119, 1600382 (2017).

11. Moodley, R., Kindness, A. \& Jonnalagadda, S.B. Elemental composition and chemical characteristics of five edible nuts (almond, Brazil, pecan, macadamia and walnut) consumed in Southern Africa. Journal of Environmental Science and Health, Part B 42, 585-591 (2007).

12. Navarro, S.L. \& Rodrigues, C.E. Macadamia Oil Extraction With Alcoholic Solvents: Yield and Composition of Macadamia Oil and Production of Protein Concentrates From Defatted Meal. European journal of lipid science and technology 120, 1800092 (2018).

13. Garg, M.L., Blake, R.J. \& Wills, R.B. Macadamia nut consumption lowers plasma total and LDL cholesterol levels in hypercholesterolemic men. The Journal of nutrition 133, 1060-1063 (2003).

14. Garg, M.L., Blake, R.J., Wills, R.B. \& Clayton, E.H. Macadamia nut consumption modulates 
favourably risk factors for coronary artery disease in hypercholesterolemic subjects. Lipids $\mathbf{4 2}$, 583-587 (2007).

15. Powell, M., Accad, A. \& Shapcott, A. Where they are, why they are there, and where they are going: using niche models to assess impacts of disturbance on the distribution of three endemic rare subtropical rainforest trees of Macadamia (Proteaceae) species. Australian Journal of Botany 62, 322-334 (2014).

16. Trueman, S. \& Turnbull, C. Effects of cross-pollination and flower removal on fruit set in Macadamia. Annals of Botany 73, 23-32 (1994).

17. Chagné, D. in Advances in botanical research, Vol. 74 1-37 (Elsevier, 2015).

18. Nock, C.J. et al. Genome and transcriptome sequencing characterises the gene space of Macadamia integrifolia (Proteaceae). BMC genomics 17, 937 (2016).

19. O’Connor, K., Hayes, B. \& Topp, B. Prospects for increasing yield in macadamia using component traits and genomics. Tree genetics \& genomes 14, 7 (2018).

20. Nock, C.J., Baten, A. \& King, G.J. Complete chloroplast genome of Macadamia integrifolia confirms the position of the Gondwanan early-diverging eudicot family Proteaceae. BMC genomics 15, S13 (2014).

21. Liu, J., Niu, Y.-F., Ni, S.-B., He, X.-Y. \& Shi, C. Complete chloroplast genome of a subtropical fruit tree Macadamia ternifolia (Proteaceae). Mitochondrial DNA Part B 2, 738-739 (2017).

22. Liu, J. et al. The whole chloroplast genome sequence of Macadamia tetraphylla (Proteaceae). Mitochondrial DNA Part B 3, 1276-1277 (2018).

23. Pisanu, P.C., Gross, C.L. \& Flood, L. Reproduction in wild populations of the threatened tree Macadamia tetraphylla: interpopulation pollen enriches fecundity in a declining species. Biotropica 41, 391-398 (2009).

24. Doležel, J., Greilhuber, J. \& Suda, J. Estimation of nuclear DNA content in plants using flow cytometry. Nature protocols 2, 2233 (2007).

25. Marçais, G. \& Kingsford, C. A fast, lock-free approach for efficient parallel counting of occurrences of k-mers. Bioinformatics 27, 764-770 (2011).

26. Vurture, G.W. et al. GenomeScope: fast reference-free genome profiling from short reads. Bioinformatics 33, 2202-2204 (2017).

27. Koren, S. et al. Canu: scalable and accurate long-read assembly via adaptive k-mer weighting and repeat separation. Genome research 27, 722-736 (2017).

28. Ruan, J. \& Li, H. Fast and accurate long-read assembly with wtdbg2. BioRxiv, 530972 (2019).

29. Walker, B.J. et al. Pilon: an integrated tool for comprehensive microbial variant detection and genome assembly improvement. PloS one 9, e112963 (2014).

30. Benson, G. Tandem repeats finder: a program to analyze DNA sequences. Nucleic acids research $\mathbf{2 7}$, 573-580 (1999).

31. Bao, W., Kojima, K.K. \& Kohany, O. Repbase Update, a database of repetitive elements in eukaryotic 
genomes. Mobile Dna 6, 11 (2015).

32. Tarailo - Graovac, M. \& Chen, N. Using RepeatMasker to identify repetitive elements in genomic sequences. Current protocols in bioinformatics 25, 4.10. 11-14.10. 14 (2009).

33. Price, A.L., Jones, N.C. \& Pevzner, P.A. De novo identification of repeat families in large genomes. Bioinformatics 21, i351-i358 (2005).

34. Edgar, R.C. \& Myers, E.W. PILER: identification and classification of genomic repeats. Bioinformatics 21, i152-i158 (2005).

35. Thiel, T., Michalek, W., Varshney, R. \& Graner, A. Exploiting EST databases for the development and characterization of gene-derived SSR-markers in barley (Hordeum vulgare L.). Theoretical and applied genetics 106, 411-422 (2003).

36. Schattner, P., Brooks, A.N. \& Lowe, T.M. The tRNAscan-SE, snoscan and snoGPS web servers for the detection of tRNAs and snoRNAs. Nucleic acids research 33, W686-W689 (2005).

37. Lagesen, K. et al. RNAmmer: consistent and rapid annotation of ribosomal RNA genes. Nucleic acids research 35, 3100-3108 (2007).

38. Nawrocki, E.P., Kolbe, D.L. \& Eddy, S.R. Infernal 1.0: inference of RNA alignments. Bioinformatics 25, 1335-1337 (2009).

39. Griffiths-Jones, S. et al. Rfam: annotating non-coding RNAs in complete genomes. Nucleic acids research 33, D121-D124 (2005).

40. Lowe, T.M. \& Eddy, S.R. A computational screen for methylation guide snoRNAs in yeast. Science 283, 1168-1171 (1999).

41. Mario, S., Rasmus, S., Stephan, W. \& Burkhard, M. AUGUSTUS: a web server for gene finding in eukaryotes. Nucleic Acids Research 32, 309-312 (2004).

42. Korf, I. Gene finding in novel genomes. BMC bioinformatics 5, 59 (2004).

43. Initiative, A.G. Analysis of the genome sequence of the flowering plant Arabidopsis thaliana. nature 408, 796 (2000).

44. Velasco, R. et al. The genome of the domesticated apple (Malus $\times$ domestica Borkh.). Nature genetics 42, 833 (2010).

45. Ming, R. et al. Genome of the long-living sacred lotus. Nelumbo nucifera.

46. Saint-Oyant, L.H. et al. A high-quality genome sequence of Rosa chinensis to elucidate ornamental traits. Nature plants 4, 473 (2018).

47. She, R., Chu, J.S.-C., Wang, K., Pei, J. \& Chen, N. GenBlastA: enabling BLAST to identify homologous gene sequences. Genome research 19, 143-149 (2009).

48. Birney, E., Clamp, M. \& Durbin, R. GeneWise and genomewise. Genome research 14, 988-995 (2004).

49. Haas, B.J. et al. Improving the Arabidopsis genome annotation using maximal transcript alignment assemblies. Nucleic acids research 31, 5654-5666 (2003).

50. Haas, B.J. et al. Automated eukaryotic gene structure annotation using EVidenceModeler and the 
Program to Assemble Spliced Alignments. Genome biology 9, R7 (2008).

51. Bairoch, A. \& Apweiler, R. The SWISS-PROT protein sequence database and its supplement TrEMBL in 2000. Nucleic acids research 28, 45-48 (2000).

52. Yuki, M., Masumi, I., Shujiro, O., Yoshizawa, A.C. \& Minoru, K. KAAS: an automatic genome annotation and pathway reconstruction server. Nucleic Acids Research 35, 182-185 (2007).

53. Jones, P. et al. InterProScan 5: genome-scale protein function classification. Bioinformatics 30, 1236-1240 (2014).

54. Bolger, A.M., Marc, L. \& Bjoern, U. Trimmomatic: a flexible trimmer for Illumina sequence data. Bioinformatics 30, 2114-2120 (2014).

55. Langmead, B. \& Salzberg, S.L. Fast gapped-read alignment with Bowtie 2. Nature methods $\mathbf{9}, 357$ (2012).

56. Simão, F.A., Waterhouse, R.M., Ioannidis, P., Kriventseva, E.V. \& Zdobnov, E.M. BUSCO: assessing genome assembly and annotation completeness with single-copy orthologs. Bioinformatics 31, 3210-3212 (2015).

57. Daehwan, K., Ben, L. \& Salzberg, S.L. HISAT: a fast spliced aligner with low memory requirements. Nature Methods 12, 357-360 (2015).

\section{Acknowledgements}

This work was supported by the National Natural Science Foundation of China (No. 31760215), the Technology Innovation Talents Project of Yunnan Province (2018HB086), and Sci-Tech Innovation System Construction for Tropical Crops Grant of Yunnan Province (RF2019/RF2020).

\section{Author Contributions}

Y.-F.N. and J.L. conceived the study and managed the project. G.-H.L., S.-B.N., and X.-Y.H. designed the scientific objectives. C.Z., Z.-Y.L., L.-D.G., and G.-H. K.collected the samples and extracted the genomic DNA. J.L. estimated the genome size, assembled the genome and carried out the gene annotation. Y.-F.N. wrote the manuscript, and all authors contributed to writing and editing the final manuscript.

\section{Additional Information}

Competing interests: The authors declare no competing interests. 